\title{
Unbiased Disagreement in Financial Markets, Waves of Pessimism and the Risk-Return Tradeoff*
}

\author{
Elyes Jouini $† \quad$ Clotilde Napp $\ddagger$
}

December 2, 2009

\begin{abstract}
Can investors with irrational beliefs be neglected as long as they are rational on average ? Do their trades cancel out with no consequences on prices, as implicitly assumed by traditional models? We consider a model with irrational investors, who are rational on average. We obtain waves of pessimism and optimism that lead to countercyclical market prices of risk and procyclical risk-free rates. The variance of the state price density is greatly increased. The long run risk-return relation is modified; in particular, the long run market price of risk might be higher than both the instantaneous and the rational ones.
\end{abstract}

${ }^{*}$ The financial support of the GIP ANR (Croyances project) and of the Risk Foundation (Groupama Chair) is gratefully ackowledged by the authors. The authors wish to thank the participants at the Swiss Banking Institute and NCCR FINRISK Seminar, at the Nomura Seminar (Mathematical Institute of the University of Oxford) and at the Stochastic Analysis and Stochastics of Financial Markets Joint Seminar Humboldt University and TU Berlin for their remarks and suggestions.

†Université Paris Dauphine, jouini@ceremade.dauphine.fr, + 33144054226

${ }^{\ddagger}$ CNRS and Université Paris-Dauphine, clotilde.napp@dauphine.fr, + 33144054642 


\title{
Unbiased Disagreement in Financial Markets, Waves of Pessimism and the Risk-Return Tradeoff
}

\begin{abstract}
Can investors with irrational beliefs be neglected as long as they are rational on average? Do their trades cancel out with no consequences on prices, as implicitly assumed by traditional models? We consider a model with irrational investors, who are rational on average. We obtain waves of pessimism and optimism that lead to countercyclical market prices of risk and procyclical risk-free rates. The variance of the state price density is greatly increased. The long run risk-return relation is modified; in particular, the long run market price of risk might be higher than both the instantaneous and the rational ones.
\end{abstract}

\section{Introduction}

Most neoclassical asset pricing models rely on the assumption that market participants are rational and maximize their expected utility under the true probabilities of uncertain economic states. There is however mounting evidence for the presence of traders with biased beliefs in the markets. How does the presence of these participants affect the behavior of financial markets ?

It is widely argued that the presence of these traders can be neglected. The first main argument is based on the work of Milton Friedman (1953), and asserts that irrational traders need not be considered since they will be eliminated in the long run. Indeed, their trading on wrong beliefs leads them to lose their wealth, and since these traders don't survive, they cannot influence the long run behavior of financial markets. The second main argument for neglecting behavioral participants relies on the fact that they should be rational on average; there is no reason for a specific systematic bias, hence investors expectations should be on 
average correct, their trades should cancel out, and there should be no impact on financial markets. The third main argument relies on an arbitrage-like argument; the actions of the rational investors should offset the actions of the irrational ones. Prices should induce rational investors (in aggregate) to overweight (relative to market weights) the assets underweighted by the irrational investors due to their erroneous beliefs, and to underweight the assets overweighted by the irrational, thereby offsetting the price effects of the irrational investors.

There is an important body of recent literature questioning the first argument. In a general equilibrium setting, Sandroni (2000) and Blume and Easley (2006) show that with intermediate consumption irrational traders do not survive in the long run. Yan (2008a) shows that only the trader with the lowest "survival index" (a function of belief accuracy, patience and risk aversion parameters) survives in the long run. In particular, if investors have the same preferences (or if their preferences are independent from their beliefs) and if at least one agent is rational, then those with incorrect beliefs cannot survive in the long run. However, the selection process is very slow. Furthermore there are two limits to the first argument. On the one hand, Kogan et al. (2006, 2008) show that survival and price impact are two different concepts. On the other hand, several agents with different beliefs may survive as in Blume and Easley (2009). The aim of our paper is then to question the second argument: can investors with irrational beliefs be neglected as long as they are rational on average ? The last section also sheds some light on the third argument.

We consider an equilibrium model with two groups of behavioral investors who are on average rational and we analyse to what extent these behavioral investors cancel out or otherwise impact equilibrium characteristics. We find that this model shares some similarities with the standard rational model. In particular, in our model, no investor gets eliminated in the long run, since no investor (or group of investors) is more wrong than any others. All the agents survive. As in the rational setting, the consumption shares of the agents remain equally distributed at all dates, which means that none of the agents wins. Finally, the market price of risk and the risk free rate are on average (over the states of the world) given 
by the market price of risk and the risk free rate of the rational setting.

However, the features of the setting with disagreement are very different from the features of the rational setting. The economy is dominated by the pessimistic agent(s) in bad states of the world and by the optimistic agent(s) in good states of the world. There are then waves of pessimism and optimism in the economy, a "pessimistic wave" in bad states of the world and an "optimistic wave" in good states of the world. Since pessimism (resp. optimism) is associated to a higher (resp. lower) market price of risk, we obtain a market price of risk that is high (low) in bad (good) states of the world. Note that this result is consistent with observed countercyclical variations in the equity premium. Indeed, there is evidence that the equity premium is time varying and as underlined by Campbell and Cochrane (1999) "equity risk premia seem to be higher at business cycles troughs than they are at peaks". For analogous reasons, we obtain that the risk free rate is lower during recessions and higher during expansions. This is consistent with observed behavior since empirical studies have confirmed that the short term rate is a procyclical indicator of economic activity (see e.g. Friedman, 1986, Blanchard and Watson, 1986). Moreover, in our model, both the market price of risk and the risk free rate exhibit mean reversion, which is consistent with the findings of, e.g., Fama and French (1988).

We also obtain specific features for long term returns. The discount rate (i.e. the rate of a zero coupon bond) converges to the "pessimistic" discount rate, i.e. the discount rate that would prevail if the pessimistic agents had the whole endowment of the economy. This result holds even though the instantaneous risk free rate is at all times given by the rational rate on average (over the states of the world). This is due to the fact that as maturity increases, the zero coupon bond becomes more and more desirable as a hedging instrument against very bad states of the world for the pessimistic agents, who then exert stronger influence on its equilibrium prices. As far as risky assets are concerned, we find that, when the volatility is not too high, the expected rolled over return (i.e. the expected return of investing one Euro in the asset and rolling it over) converges to the return that would prevail in the economy 
populated by the more optimistic agents only. This is due to the fact that the optimistic agents overestimate the expected return of the risky asset and when the volatility is not too high, holding the risky asset between time 0 and a given time in the far future is more attractive for them, as a hedging instrument against endowment risk. The long term return is then higher than in the standard rational setting and higher than the instantaneous return. The long term risk premium is also higher than the rational long term risk premium and than the instantaneous risk premium. It is higher than both the long term risk premia in the optimistic and in the pessimistic economy ${ }^{1}$. In other words, the presence of irrational traders modifies the long term relation between risk and return and introduces a distortion between the long term and the short term risk-return tradeoff.

We obtain that disagreement (with aggregate rationality) induces more variance on the state price density than the rational setting, which is interesting in relation with Hansen and Jagannathan $(1991,1997)$. The state price density is no longer lognormal ${ }^{2}$. This is consistent with the empirical literature on the state price density extracted from assets prices (see, e.g. Jackwerth and Rubinstein, 1996, or Aït-Sahalia and Lo, 1998).

Our main model considers the setting with two groups of agents, logarithmic utility functions, and constant belief parameters. We analyse the robustness of our results to other specifications of the utility functions. For general power utility functions, we show that our results remain essentially true. We also obtain additional results on the volatility of the stock whose dividends are given by the aggregate endowment, which is fluctuating and whose level can deviate from the standard rational level. On the contrary, we show that our results do not hold for exponential utility functions. Indeed, the waves of optimism and pessimism result from the fluctuations in the (relative) levels of absolute risk tolerances (which are given by the consumption shares in the case of power or logarithmic utility functions). For CARA utility functions the relative levels of risk tolerance do not fluctuate. In particular, we obtain

\footnotetext{
${ }^{1}$ Whereas the instantaneous risk premium is an average of the optimistic and of the pessimistic risk premia and is on average given by the rational instantaneous rate.

${ }^{2}$ It is a mixture of lognormal distributions.
} 
that the market price of risk is constant and given by the standard rational market price of risk $^{3}$. We also consider more complex forms of disagreement, and in particular we consider the case where the investors may switch from pessimism to optimism and conversely. We show that our results pertain as long as there is some persistence in optimism and pessimism. Moreover, in general models of disagreement for which there is not necessarily a persistence in the individual biases, we show that we obtain results that are locally of the same nature. We finally analyse if our results are robust to the presence of a third group of rational investors. Apart from the survival issues, the results remain qualitatively the same. This provides an answer to the third main argument presented above for neglecting behavioral investors. Offsetting actions by rational investors do not typically suffice to cause the price effects of wrong beliefs to disappear. This conclusion, drawn from Fama and French (2008), is extended here to the case of unbiased beliefs.

Levy et al. (2006), Duchin and Levy (2009) and Yan (2008b) are also interested in the impact of "unbiased" disagreement on prices or more generally on equilibrium characteristics and are closely related to our work. Levy et al. (2006) show that if investors have heterogeneous but unbiased beliefs about the expected returns, the homogeneous CAPM pricing holds. Duchin and Levy (2009) analyse the impact of disagreement on the return variances in a mean-variance setting. More precisely, their paper shows that contrary to the disagreement on the mean, unbiased disagreement on the variance has systematic pricing effects. Yan (2008b) analyses the impact of independent biases in investors demand functions on assets prices. He first shows that independent biases affect prices if investors demand functions are non linear functions of the bias (as in the case studied by Duchin and Levy, 2009). Then he shows in a two period setting that, even if the demand function is linear in the bias, the fluctuation of the wealth distribution leads to stock return negative autocorrelation. Our paper is the first to analyse the dynamics as well as the long run properties of unbiased disagreement models.

\footnotetext{
${ }^{3}$ This case is essentially the continuous time analog of Levy et al. (2006).
} 
Related papers also include Abel (1989), Cabrales and Hoshi (1996), Calvet et al. (2002), Detemple and Murthy (1994), Zapatero (1998), Berrada (2006), Jouini and Napp (2006, 2007), and Gollier (2007), all of which deal with the equilibrium characteristics in a heterogeneous belief framework. Our paper is to be contrasted to the works of Scheinkman and Xiong (2003), Dumas et al. (2009), Li (2007) and David (2008) who all consider specific models of belief divergence and updating, while our aim is to explore the impact of "noisy" beliefs, independently of a specific dynamics for belief formation. Our paper is also to be contrasted to the strand of disagreement literature in which investors learn from prices as in e.g., Admati (1985), Biais et al. (2009) and DeMarzo and Skiadas (1998).

All proofs are in the Appendices (Appendix I for the main model, Appendix II for the extensions).

\section{The model}

We consider a continuous-time pure exchange Arrow-Debreu economy, with a single consumption good and two risk averse agents (or groups of agents) trying to maximize their expected utility from future consumption. We assume that both agents have the same utility function for consumption and the same time preference rate, but can differ in their subjective beliefs about the future of the economy. A filtered probability space $\left(\Omega, F,\left(F_{t}\right), P\right)$ describing uncertainty is given and each agent has a von Neumann Morgenstern utility for future consumption of the form

$$
U_{i}(c)=E^{Q_{i}}\left[\int_{0}^{\infty} \exp (-\rho t) u\left(c_{t}\right) d t\right]
$$

where $\rho$ represents the time preference rate parameter, $u$ is the common utility function, and the subjective belief of each agent is represented by the subjective probability measure $Q_{i}$, equivalent to the initial probability $P$. We let $M^{i}$ denote the density of $Q_{i}$ with

respect to $P$, i.e., $\frac{d Q_{i}}{d P}=M^{i}$. Letting $\left(M_{t}^{i}\right)$ denote the density process $M_{t}^{i} \equiv E\left[M^{i} \mid F_{t}\right]$, 
the utility of agent $i$ for the consumption stream $c$ can be written in the form $U_{i}(c)=$ $E\left[\int_{0}^{\infty} M_{t}^{i} \exp (-\rho t) u\left(c_{t}\right) d t\right]$.

We let $e^{*}$ denote the aggregate endowment process in the economy. We make the assumption that the processes $e^{*}$ and $M^{i}$ satisfy the following stochastic differential equations

$$
\left\{\begin{array}{c}
d e_{t}^{*}=\mu e_{t}^{*} d t+\sigma e_{t}^{*} d W_{t} \\
d M_{t}^{i}=\delta_{i} M_{t}^{i} d W_{t}
\end{array}\right.
$$

where $W$ is a standard unidimensional ${ }^{4}\left(\left(F_{t}\right), P\right)$-Brownian motion, $(\mu, \sigma) \in\left(\mathbb{R}_{+}\right)^{2}$ are given constants and $\delta_{i}$ is a stochastic process $\left(\delta_{i}(t, \omega)\right)$. This is roughly the most general model of belief divergence in a diffusion setting ${ }^{5}$. The assumption on $e^{*}$ means that $e^{*}$ is a geometric Brownian motion with drift. In such a context, Agent 1 and Agent 2 both know that the volatility parameter is given by $\sigma$ but have different beliefs about the constant growth rate $\mu$. By Girsanov Theorem, we have

$$
\left\{\begin{array}{l}
d e_{t}^{*}=\left(\mu+\sigma \delta_{1}\right) e_{t}^{*} d t+\sigma e_{t}^{*} d W_{t}^{1} \\
d e_{t}^{*}=\left(\mu+\sigma \delta_{2}\right) e_{t}^{*} d t+\sigma e_{t}^{*} d W_{t}^{2}
\end{array}\right.
$$

where for $i=1,2, W_{t}^{i} \equiv W_{t}-\delta_{i} t$ is a Brownian motion under $Q_{i}$, which means that Agent 1 believes that the aggregate endowment growth rate is given by $\mu_{1} \equiv \mu+\sigma \delta_{1}$, whereas Agent 2 believes that it is given by $\mu_{2} \equiv \mu+\sigma \delta_{2}$ and both agents agree on the volatility parameter $\sigma$. The parameter $\delta_{i}=\frac{\mu_{i}-\mu}{\sigma}$ then measures investor $i$ 's error in his perceived economic growth (normalized by the level of risk) $^{6}$. In this model, an agent is irrational at time $t$ and in state

\footnotetext{
${ }^{4}$ For the sake of simplicity, we only consider one source of risk. The results can be generalized to multidimensional sources of risk.

${ }^{5}$ Indeed, if $M^{i}$ is a positive martingale process, the fact that it can be written in the form $d M_{t}^{i}=\delta_{i} M_{t}^{i} d W_{t}$ for some adapted process $\left(\delta_{i}\right)$ is then just a regularity assumption.

${ }^{6}$ The fact that both agents agree on the volatility parameter is implied by the assumption that the individual probabilities $Q_{i}$ are equivalent to the initial one $P$. This assumption is quite natural. Note that if the $Q_{i}$ were absolutely continuous with respect to $P$ and not equivalent, and if there existed an event $A$ with a positive probability for Agent 1 and a zero probability for Agent 2, equilibrium could not be reached since the demand of Agent 1 would be infinite in event A. Moreover, as already noticed by Basak (2000), or Yan (2008a), this parametrization is consistent with the insight from Merton (1980) that the expected
} 
$\omega$, if $\delta_{i}(t, \omega) \neq 0$, optimistic if $\delta_{i}(t, \omega)>0$ and pessimistic if $\delta_{i}(t, \omega)<0$.

We assume that the agents are on average rational, i.e. that $\delta_{1}(t, \omega)=\delta(t, \omega)$ and $\delta_{2}(t, \omega)=-\delta(t, \omega)$. We also assume that individual endowments denoted by $e^{*^{i}}$ are the same with $e^{*^{i}}=\frac{1}{2} e^{*}$. This amounts to assuming that at each date and state of the world, beliefs and wealth are independent and implies that both the ex-ante unweighted and the ex-ante wealth-weighted average beliefs are rational.

An Arrow-Debreu equilibrium relative to the beliefs $M^{i}$ is defined by a positive density price process $q^{*}$ and a pair of optimal consumption plans $\left(c_{i}^{*}\right)_{i=1,2}$ such that markets clear, i.e.,

$$
\left\{\begin{array}{c}
c_{i}^{*}=c_{i}\left(q^{*}, M^{i}, e^{*^{i}}\right) \\
\sum_{i=1}^{2} c_{i}^{*}=e^{*}
\end{array}\right.
$$

where $c_{i}(q, M, e) \equiv \arg \max _{E\left[\int_{0}^{T} q_{t}\left(c_{t}-e_{t}\right) d t\right] \leq 0} E\left[\int_{0}^{T} M_{t} \exp (-\rho t) u\left(c_{t}\right) d t\right]$.

In order to deal with asset pricing issues, we suppose that agents can continuously trade in a riskless asset and in risky stocks. We let $S^{0}$ denote the riskless asset price process with dynamics $d S_{t}^{0}=r_{t}^{f} S_{t}^{0} d t$. Since there is only one source of risk, all risky assets have the same instantaneous Sharpe ratio and it suffices to focus on one specific risky asset. In order to better analyse the instantaneous as well as the long term risk return tradeoff, we consider a risky asset $S$ with given volatility level ${ }^{7} \sigma_{s}$, and with dynamics $d S_{t}=S_{t}\left[\mu_{s} d t+\sigma_{s} d W_{t}\right]$. The risk free rate process $r^{f}$, as well as the stock return drift $\mu_{s}$ are to be determined endogenously in equilibrium.

\section{Constant belief parameters and log-utility functions}

In this section we consider constant belief parameters $\delta_{i}$. This setting is the most simple and natural extension of a standard rational model where all agents know that the true growth rate is a constant $\mu$ to the case with disagreement (with on average rational beliefs);

return is harder to estimate than the variance.

${ }^{7}$ Note that by market completeness such an asset with a given volatility level always exists. 
one agent believes that the growth rate is $\mu+\sigma \delta$ and the other believes that it is $\mu-$ $\sigma \delta$. The restriction implied by such a modelling is that one group of agents systematically overestimates the growth rate while the other group of agents systematically underestimates it. This restriction is consistent with the interpretation of the bias on the beliefs as a behavioral bias characterising the behavior of the individual towards risk, like the individual distorsions of the underlying probability distributions, introduced in the recent decision theory literature. With such an interpretation, an individual is more or less pessimistic in the same way as she is more or less risk tolerant or impatient ${ }^{8}$. The choice of constant parameters can also model "tastes for assets" as in e.g. Fama and French (2008). In this case, a positive $\delta$ would correspond to the agents who like the asset and a negative $\delta$ to the agents who dislike the asset. For simplicity we start by considering logarithmic utility functions ${ }^{9}$. We analyze in the next section more general belief parameters and utility functions.

In such a setting, it is easy to obtain that there exists a unique equilibrium given by

$$
\begin{aligned}
& c_{1}^{*}=\left(\frac{M^{1}}{M^{1}+M^{2}}\right) e^{*}, \quad c_{2}^{*}=\left(\frac{M^{2}}{M^{1}+M^{2}}\right) e^{*}, \\
& q^{*}=\left(\frac{1}{2} M^{1}+\frac{1}{2} M^{2}\right) \exp (-\rho t)\left(\frac{1}{e^{*}}\right) .
\end{aligned}
$$

We let $\tau \equiv \tau_{1} \equiv \frac{c_{1}^{*}}{e^{*}}$ denote the consumption share of agent 1 . The consumption share of agent 2 is then given by $\tau_{2} \equiv 1-\tau \equiv \frac{c_{2}^{*}}{e^{*}}$. Note that $\tau_{i}$ also corresponds to the individual relative level of absolute risk tolerance given by $-\frac{u^{\prime}\left(c_{i}^{*}\right)}{u^{\prime \prime}\left(c_{i}^{*}\right)}\left[\sum_{i=1}^{2}-\frac{u^{\prime}\left(c_{i}^{*}\right)}{u^{\prime \prime}\left(c_{i}^{*}\right)}\right]^{-1}$.

In the standard rational case, the consumption share of each agent is time and state

\footnotetext{
${ }^{8}$ If the bias corresponds to a behavioral bias having decision theoretical foundations, then it is consistent to suppose that the bias is persistent, one group of agents remaining optimistic and the other group of agents remaining pessimistic. Our notion of optimism/pessimism coincides in our setting with the notions of optimism/pessimism adopted by e.g., Yaari (1987), Chateauneuf and Cohen (1994) and Dieciedue and Wakker (2001). Chateauneuf and Cohen (1994) relate it to the notion of First Stochastic Dominance, while Yaari (1987) and Diecidue and Wakker (2001) relate it to the notion of Monotone Likelihood Ratio. These notions coincide in our setting.

${ }^{9}$ Note that, in the logarithmic setting, the price of the stock whose dividends are the aggregate endowment is given by $S_{t}=\frac{1}{\rho} e_{t}^{*}$, hence there is no impact of unbiased disagreement on the price of this stock. This is not true in the general power utility setting.
} 
independent and equal to $1 / 2$. In the case with one rational and one irrational agent, we know (see e.g. Kogan et al., 2006) that the irrational agent becomes extinct in the sense that her consumption share converges to 0 and that the consumption share of the rational agent converges to 1 (almost surely). More generally, if there is a bias on average, one agent being more wrong than the other, the more rational agent "wins" in the very long run: when $\left|\delta_{1}\right|>\left|\delta_{2}\right|$ then $\tau_{1}(t)$ converges to 0 and $\tau_{2}(t)$ converges to 1 almost surely (see, e.g., Yan, 2008a). The economy ends up being dominated by the more rational agent. As expected, the situation with no bias on average is very different.

\section{Proposition 3.1. Distribution of Consumption Shares}

1. For all time $t$, the random variable $\tau(t)$ is symmetric with respect to $\frac{1}{2}$.

2. Each agent has a larger share of aggregate endowment in the states that she thinks more probable. The consumption share $\tau\left(W_{t}\right)$ is an increasing function of the Brownian motion $W_{t}$, with $\lim _{W \rightarrow 0} \tau(W)=0$ and $\lim _{W \rightarrow \infty} \tau(W)=1$.

3. The stochastic process $\tau(t)$ exhibits mean-reversion and satisfies the following Stochastic Differential Equation

$$
d \tau(t)=2 \delta \tau(1-\tau)\left[\delta(1-2 \tau) d t+d W_{t}\right]
$$

Proposition 3.1 implies in particular that the consumption shares of both agents are on average (over the states of the world) given by the standard rational consumption shares. None of the agents "wins". At all times, consumption is equally shared in the sense that the consumption shares of the agents are identically distributed. Both agents survive in the long run. These results are due to the fact that no agent is more wrong than the other ${ }^{10}$.

However, we obtain that at the equilibrium, each agent has a larger share of aggregate consumption in the states that she thinks more probable, which is intuitive. These are

\footnotetext{
${ }^{10}$ In Yan (2008a)'s terminology, the agents have the same survival index.
} 
the good states of the world for the optimistic agent and the bad states of the world for the pessimistic agent. This implies that the consumption shares are biased in favor of the optimistic agents in the good states of the world, and in favor of the pessimistic agents in the bad states of the world. Moreover, for very good (resp. very bad) states of the world, the optimistic agents (resp. pessimistic) dominate the economy, i.e. their consumption share is near one. The average belief fluctuates then between the optimistic and the pessimistic ones and there are waves of optimism and pessimism as in fads models (Cochrane, 1991).

The main content of Point 3 is the mean reversion property. Even though each consumption share either converges to 0 or 1 asymptotically along each trajectory, consumption shares have a tendency to revert to their average level of $1 / 2$. The reason for the mean reversion of consumption shares is the following. We know by Euler Equations that at the equilibrium the marginal utilities are equal at each time and state of the world, i.e. $M^{1} u^{\prime}\left(c_{1}^{*}\right)=M^{2} u^{\prime}\left(c_{2}^{*}\right)$. Consider a time and state of the world with a high consumption share (i.e. above average) for the optimistic agent $c_{1}^{*}>c_{2}^{*}$. Then $u^{\prime \prime}\left(c_{1}^{*}\right)>u^{\prime \prime}\left(c_{2}^{*}\right)$ and an increase in aggregate endowment will more favour Agent 2 than Agent 1, thereby reducing the consumption share of the optimistic agent, which tends to revert to its average level of $1 / 2$.

Note that the individual consumption shares $\tau_{i}$ also correspond to the individual wealth shares of agent $i$, given by $E\left[\int_{t}^{+\infty} q_{s}^{*} c_{i}^{*}(s) d s\right]\left[E\left[\int_{t}^{+\infty} q_{s}^{*} e_{s}^{*} d s\right]\right]^{-1}$, so that all the results obtained on the instantaneous consumption shares also hold for the wealth shares in the log setting.

\subsection{Risk-free rate and market price of risk}

The fact that the consumption shares or the wealth shares (which represent the relative levels of risk tolerance) fluctuate in time and in state of the world has an impact on asset pricing, that we now analyze. We recall that in the standard rational setting the risk free rate and the market price of risk $\left(M P R \equiv \frac{\mu_{s}-r^{f}}{\sigma_{s}}\right)$ are time and state independent and given by $r^{f}($ stdd $)=\rho+\mu-\sigma^{2}$ and $M P R($ stdd $)=\sigma$. 
Proposition 3.2. Risk free rate and market price of risk

1. The risk free rate and the market price of risk are given by

$$
\begin{aligned}
r_{t}^{f} & =r^{f}(\text { stdd })+\sigma\left(\tau_{1}(t)-\tau_{2}(t)\right) \delta \\
M P R_{t} & =M P R(\text { stdd })-\left(\tau_{1}(t)-\tau_{2}(t)\right) \delta,
\end{aligned}
$$

2. The market price of risk is countercyclical: it is a decreasing function of the Brownian motion $W$ that governs aggregate endowment.

3. The risk free rate is procyclical: it is an increasing function of $W$.

4. The risk free rate and market price of risk stochastic processes exhibit mean reversion. They satisfy the following Stochastic Differential Equations

$$
\begin{aligned}
d M P R_{t} & =-4 \delta^{2} \tau_{1}(t) \tau_{2}(t)\left[\left(M P R_{t}-E\left[M P R_{t}\right]\right) d t+d W_{t}\right] \\
d r_{t}^{f} & =-4 \delta^{2} \tau_{1}(t) \tau_{2}(t)\left[\left(r_{t}^{f}-E\left[r_{t}^{f}\right]\right) d t-\sigma d W_{t}\right]
\end{aligned}
$$

The distributions of the market price of risk and of the risk free rate are symmetric with respect to the standard quantities, and we retrieve on average over the states of the world the standard market price of risk and the standard risk free rate, which is consistent with the fact that our agents are on average rational. However, the behavior of the risk free rate and of the market price of risk is inherited from the behavior of the consumption shares (or the risk tolerances). Letting $r_{t}^{i, f}$ and $M P R^{i}$ denote the risk free rate and the market price of risk that would prevail if Agent $i$ had all the endowment, we easily obtain that the risk free rate and the market price of risk lie inside the range bounded by the two limiting cases $^{11}$, i.e. $r^{f} \in\left[r^{2, f}, r^{1, f}\right]$ and $M P R \in\left[M P R^{1}, M P R^{2}\right]$. Since we know that the consumption shares are biased in favor of the optimistic agents (resp. pessimistic agents) in good states

\footnotetext{
${ }^{11}$ In the case of power utility functions (see the next section), the result on the market price of risk remains valid, i.e., $M P R \in\left[M P R^{1}, M P R^{2}\right]$ but the risk free rate can lie outside the interval $\left[r^{2, f}, r^{1, f}\right]$.
} 
of the world (resp. bad states of the world), we obtain that the risk free rate and the market price of risk are biased in favor of the risk free rate and the market price of risk of the optimistic (resp. pessimistic) agents in good states of the world (resp. bad states of the world). Moreover, positive (resp. negative) shocks lead to an increase of the weight of the optimistic (resp. pessimistic) agents. This leads to the results of Points 2 and 3. The market price of risk is countercyclical. This result is consistent with the observed variations of the equity premium. Indeed, there is evidence that the equity premium is time varying and as underlined by, e.g., Campbell and Cochrane (1999) "equity risk premia seem to be higher at business cycles troughs than they are at peaks". For analogous reasons, we obtain that the risk free rate is lower during recessions and higher during expansions. This is also consistent with observed behavior since empirical studies have confirmed that the short term rate is a procyclical indicator of economic activity (see e.g. Friedman, 1986, Blanchard and Watson, 1986). Moreover, we obtain in Point 4 that the market price of risk and the risk free rate exhibit mean reversion.

\subsection{State price density moments}

We know that in the standard rational setting, the state price density, given by $q_{s t d d}^{*}=$

$\exp (-\rho t) u^{\prime}\left(e^{*}\right)$, is lognormal with $\log q_{s t d d}^{*} \sim \mathcal{N}\left(\left(-\rho-\mu+\frac{\sigma^{2}}{2}\right) t, \sigma^{2} t\right)$. In our setting, the state price density is a weighted average of the state price densities that would prevail if one of the agents had all the endowment, i.e. $q^{*}=\frac{1}{2} q^{*^{1}}+\frac{1}{2} q^{*^{2}}$ where $q^{*^{i}}=\exp (-\rho t) M^{i} u^{\prime}\left(e^{*}\right)$. Each state price density $q^{*^{i}}$ is lognormally distributed and the state price density in our setting $q^{*}$ is a mixture of two lognormal distributions. In particular, it is not lognormal as in the standard setting, which is consistent with the empirical literature on the state price density extracted from assets prices (see, e.g. Jackwerth and Rubinstein, 1996, or Aït-Sahalia 
and Lo, 1998). Furthermore, direct computation gives us

$$
\begin{aligned}
\frac{E\left[q_{t}^{*}\right]}{E\left[q_{s t d d}^{*}(t)\right]} & =\cosh (\sigma \delta t) \geq 1 \\
\frac{\operatorname{Var}\left[q_{t}^{*}\right]}{\operatorname{Var}\left[q_{s t d d}^{*}(t)\right]} & =\frac{e^{\left(\sigma^{2}-\delta^{2}\right) t}-1+e^{\left(\delta^{2}+\sigma^{2}\right) t} \cosh (4 \sigma \delta t)-\cosh (2 \sigma \delta t)}{2\left(e^{\sigma^{2} t}-1\right)} \geq 1 .
\end{aligned}
$$

which means that disagreement increases the mean and the variance of the state price density. Numerically, the impact on the mean is very small. However, the impact on the variance is much more important. For instance, if we take $\mu=1.8 \%$ and $\sigma=3.6 \%$, as in Mehra and Prescott (1985), and $\delta=0.5$ (which means that Agent 1 anticipates a growth rate of $3.6 \%$ while Agent 2 anticipates a zero growth rate) we obtain that the variance of the state price density in the model with disagreement is 26 times the variance in the standard model $^{12}$. These results are interesting in light of Hansen and Jagannathan $(1991,1997)$ and in particular on the bounds imposed by security market data on the mean and the standard-deviation of the state price density.

In fact, as seen through Equations (3.1) and (3.2) our state price density is the same as in an equivalent homogeneous economy with aggregate endowment $e^{*}$ and with a consensus belief $M \equiv \frac{1}{2}\left(M^{1}+M^{2}\right)$ and it is easy to check that, for the consensus agent, aggregate endowment follows the dynamics

$$
d e_{t}^{*}=\left(\mu+\tau_{1} \sigma \delta-\tau_{2} \sigma \delta\right) e_{t}^{*} d t+\sigma e_{t}^{*} d W_{t}^{Q}
$$

where $W^{Q}$ is a Brownian Motion for the consensus agent. For the consensus agent, the instantaneous expected growth rate is then time varying and stochastic and evolves smoothly between the two bounds $\mu_{1}$ and $\mu_{2}$. From the consensus agent point of view, this "regime shifting model" can be seen as a smooth version of the regime switching model of e.g., David and Veronesi (2002). Moreover, Equation (3.5) implies that the consensus agent is

\footnotetext{
${ }^{12}$ With the same figures we have $\frac{E\left[q_{t}^{*}\right]}{E\left[q_{\text {stdd }}^{*}(t)\right]}=1.0006$.
} 
optimistic in good states of the world and pessimistic in bad states of the world even though there is no bias at the aggregate level since $E\left[\left(\mu+\tau_{1} \sigma \delta-\tau_{2} \sigma \delta\right)\right]=\mu$. We easily get that $E^{Q}\left[\log e_{t}^{*}\right]=t\left(\mu-\frac{1}{2} \sigma^{2}\right)$, and $\operatorname{Var}^{Q}\left[\log e_{t}^{*}\right]=\sigma^{2} t+t^{2} \sigma^{2} \delta^{2}$. From the consensus agent point of view, the mean is the same and there is then more variance on the distribution of aggregate endowment, the additional variance being the result of the drift variations. As argued by Rubinstein (1976), the variations of the state price density capture the corrections for risk in assets valuation. The additional variance of the state price density is then directly related to the additional variance at the consensus agent level.

\subsection{Long term returns}

We now turn to considerations about long term returns of riskless and risky assets. In the standard setting, for all time $T$, the rate of return associated to a zero coupon bond maturing at time $T$ is given by $R$ (stdd) $=\rho+\mu-\sigma^{2}$ and is equal to the instantaneous risk free rate. The instantaneous return for asset $S$, whose volatility level is given by $\sigma_{S}$, is constant and given by $\mu_{S}(s t d d)=\rho+\mu-\sigma^{2}+\sigma_{S} \sigma$. The expected risky asset cumulative return (i.e. the expected return of investing one Euro in the asset and rolling it over) between time 0 and time $T$ is given by $C R_{T}^{S}(s t d d) \equiv \frac{1}{T} \log E\left[S_{T}\right]=\frac{1}{T} \log E\left[\exp \left\{\left(r_{s t d d}^{f}+\sigma_{S} \sigma-\frac{\sigma_{S}^{2}}{2}\right) T+\sigma_{S} W_{T}\right\}\right]$. It is constant and given by the instantaneous return $\mu_{S}(s t d d)$.

In our setting, we have already seen that the instantaneous risk free rate is the consumption share weighted average of the instantaneous rates that would prevail if one of the agents had all the endowment (Proposition 3.2, Point 1). We easily obtain that the instantaneous return for asset $\mathrm{S}$ at time $t$ has similar properties, and is given by $\mu_{S}(t)$ $=\rho+\mu-\sigma^{2}+\sigma \sigma_{S}+\left(\tau_{1}-\tau_{2}\right) \delta\left(\sigma-\sigma_{S}\right)$. In particular it is on average equal to the rational instantaneous return $\mu_{S}(s t d d)$ and fluctuates between the two bounds. The next proposition

identifies the long run behavior of the discount rate $R_{T} \equiv-\frac{1}{T} \log E\left[q_{T}^{*}\right]$, of the cumulative return on the risky asset $C R_{T}^{S} \equiv \frac{1}{T} \log E\left[S_{T}\right]$ and of the risk premium $r p_{T}^{S} \equiv \frac{1}{T} \log E\left[S_{T}\right]-R_{T}$ in our setting. 
Proposition 3.3. $\quad$ 1. The asymptotic discount rate is given by $R_{\infty} \equiv \lim _{T} R_{T}=\rho+$ $\mu-\sigma^{2}-\delta \sigma$. It is the discount rate that would prevail with only the pessimistic agent present in the economy.

2. For an asset whose volatility is given by $0 \leq \sigma_{S} \leq \sigma$, the asymptotic cumulative return is given by $C R_{\infty}^{S} \equiv \lim _{T} C R_{T}^{S}=\mu_{S}($ stdd $)+\delta\left(\sigma-\sigma_{S}\right)$ with $\mu_{S}($ stdd $)=\rho+\mu-\sigma^{2}+\sigma \sigma_{S}$. It is the cumulative return that would prevail with only the optimistic agent present in the economy. In particular, $C R_{\infty}^{S} \geq C R^{S}\left(\right.$ stdd) and $C R_{\infty}^{S} \geq \mu_{S}(t)$ for all $t$.

3. For an asset whose volatility is given by $0 \leq \sigma_{S} \leq \sigma$, the asymptotic risk premium is given by $r p_{\infty}^{S} \equiv \lim _{T} r p_{T}^{S} \equiv \lim _{T}\left(\frac{1}{T} \log E\left[S_{T}\right]-R_{T}\right)=r p^{S}(s t d d)+\delta\left(\sigma-\sigma_{S}\right)+\delta \sigma$, where $r p^{S}($ stdd $)=\sigma \sigma_{S}$. In particular, it is higher than the standard asymptotic risk premium and higher than the instantaneous risk premium.

This means that the long run discount rate is always the pessimistic rate. We have seen in Proposition 3.2 that the risk free rate is on average given by the rational rate since for all $t, E\left[r_{t}^{f}\right]=\rho+\mu-\sigma^{2}$. We have also seen that none of the agents vanishes and that the distribution of the risk free rate is, at each date, symmetric with respect to the rational rate. However, the discount rate converges to the discount rate of the more pessimistic agent. This apparent contradiction is due to the fact that the behavior of the instantaneous risk free rate $r_{t}^{f}$ is driven by the consumption shares at time $t$. As already seen, since no agent wins, at all times the risk free rate is on average given by the rational rate. Concerning the discount rate, as maturity increases, the zero coupon bond, as a hedging instrument against very bad states of the world, becomes more and more desirable for the pessimistic agent, who then exerts stronger influence on its equilibrium price.

As far as risky assets returns are concerned, the long term cumulative return for low volatility assets is higher than the (instantaneous or long term) return in the standard setting. It is also higher than the instantaneous return and it is given by the cumulative return of the asset in an economy made of the optimistic agent only. The interpretation 
is the following. The optimistic agent overestimates the expected return of the risky asset and when the volatility is not too high (compared to the volatility of aggregate endowment), holding the risky asset between time 0 and a given time in the far future is more attractive for her, as a hedging instrument against endowment risk. The optimistic agent consequently exerts a stronger influence on its equilibrium cumulative return.

Both effects impact the risk premium. The asymptotic risk premium is higher than the standard asymptotic risk premium. It is higher than both the optimistic and the pessimistic risk premia and also higher than the instaneous risk premium. A possible interpretation is that there is in the long term an additional risk, a sentiment risk due to disagreement, that modifies the standard risk return relation.

\section{Other Utility Functions and more general belief parameters}

The aim of this section is to consider if the results of the previous section are robust to more general utility functions and/or to nonconstant belief parameters.

\subsection{Other utility functions}

We have considered in the previous section logarithmic utility functions. Consider now the more general setting of power utility functions with $u^{\prime}(x)=x^{-1 / \eta}$. We provide in the Appendix all the results in this setting and we sum up here the main conclusions. As shown in e.g. Jouini and Napp (2007), there is an aggregation bias. However, modulo this bias, the conclusions remain essentially valid. As far as survival issues are concerned, we obtain that both agents survive and there is a sort of stationarity (see Appendix, II-B). At each date, there is a pessimistic bias in bad states of the world and an optimistic bias in good states of the world (Appendix, II-C). As far as asset pricing results are concerned, we

obtain (Appendix, II-D) that the market price of risk is countercyclical and exhibits mean reversion. The risk free rate is procyclical (in "very good" and "very bad" states of the world). The discount rate is still converging to the discount rate of the pessimistic agent in 
the long run. Moreover, we obtain in the power utility setting results on the volatility of the stock whose associated dividends are the aggregate endowment (Appendix, II-E). In the myopic logarithmic setting, this volatility is necessarily given by the volatility of aggregate consumption, as in the standard rational setting. For more general power functions, it is time and state varying. In the long run, the volatility converges to the volatility of aggregate consumption. The stock price dividend ratio $\frac{S_{t}}{e_{t}^{*}}$ is also time and state varying and converges in the long run with the same probability either to the price dividend ratio of the pessimistic economy or to the price dividend ratio of the optimistic economy.

Note that our results are not robust to an exponential specification of the utility function, in particular those on asset pricing ${ }^{13}$. In fact, in the exponential setting, as in the logarithmic or power setting, the risk free rate and the market price of risk are given by the risk tolerance weighted averages of the individual risk free rates and market prices of risk (modulo the bias). In the case of exponential utility functions (CARA), the relative levels of absolute risk tolerances are constant and lead to constant market price of risk and risk free rate. We emphasize that the fluctuations in the market price of risk and in the risk free rate are due to fluctuations in the levels of risk tolerances (and not in the levels of consumptions shares, even if both notions coincide in the case of power utility functions).

\subsection{More general belief parameters}

We have considered so far a model of disagreement, in which the disagreement is on average zero but also constant in time and in states of the world, i.e. $\delta_{i} \in \mathbb{R}$. We now analyse the robustness of our results to more general specifications of disagreement, with belief parameters that are no longer constant but stochastic processes $\left(\delta_{i}(t, \omega)\right)$. We still assume that the agents are on average rational, i.e. that $\delta_{1}(t, \omega)=\delta(t, \omega)$ and $\delta_{2}(t, \omega)=-\delta(t, \omega)$.

In such a setting the consumption shares are given by $\tau_{i}(t, \omega)=\frac{M^{i}}{M^{1}+M^{2}}$, which means

\footnotetext{
${ }^{13}$ Adapting the results of Jouini and Napp (2007, Section 4.1) to the case of unbiased disagreement, we get that the market price of risk in the exponential setting is given by the standard market price of risk $M P R=M P R(s t d d)$ and the risk free rate is given by the standard risk free rate modulo a (constant) bias due to beliefs dispersion, $r^{f}=r^{f}($ stdd $)+\frac{1}{2} \delta^{2}$.
} 
that, as before, the consumption share is high in states of the world that the agent thinks more probable. The consumption share of Agent 1 is greater than the consumption share of Agent 2 in states $\omega$ that she overweights, more precisely, in states $\omega$ such that $\int_{0}^{t} \delta(s, \omega) d W_{s}>0$. Note that on average we have $E\left[\int_{0}^{t} \delta(s, \omega) d W_{s}\right]=0$. The quantity $\int_{0}^{t} \delta(s, \omega) d W_{s}$ in a way measures the degree at which nature has favoured (or disfavoured) Agent 1 with respect to Agent 2 between date 0 and date $t$. In other words there is a bias towards the agent who has been less wrong between date 0 and date $t$, given the evolution of the economy during the same period. We can show (see the Appendix) that, as in the model with constant parameters, the consumption share of Agent 1 satisfies the following SDE

$$
d \tau_{1}(t)=2 \delta \tau_{1} \tau_{2}\left[\delta\left(\tau_{2}-\tau_{1}\right) d t+d W_{t}\right]
$$

There is then "mean reversion" in the dynamics of the consumption shares, in the sense that when the consumption share is high, it has a tendency to decrease. Moreover, we have $d \log \left(\frac{\tau_{1}(t, \omega)}{\tau_{2}(t, \omega)}\right)=\delta(t, \omega) d W_{t}$, which means that there is locally a shift in favor of (against) optimistic agents following good (bad) news.

As far as asset pricing issues are concerned, it is easy to obtain that we have

$$
\begin{aligned}
r_{t}^{f} & =r^{f}(\operatorname{stdd})+\sigma \delta(t, \omega)\left[\tau_{1}(t, \omega)-\tau_{2}(t, \omega)\right] \\
M P R_{t} & =M P R(\operatorname{stdd})-\left[\tau_{1}(t, \omega)-\tau_{2}(t, \omega)\right] \delta(t, \omega) .
\end{aligned}
$$

The states of the world for which the market price of risk is high are the states of the world for which the risk free rate is low. The market price of risk (resp. the risk free rate) is lower (resp. higher) in states of the world that are "good" for the (locally) optimistic agent (in the sense that her consumption share is high). These are not necessarily the "good" states of the world, since the market price of risk is lower than the standard market price of risk when $\left(\int_{0}^{t} \delta(s, \omega) d W_{s}\right) \delta(t, \omega)>0$.

Notice that after a good shock $\left(\int_{0}^{t} \delta_{i}(s, \omega) d W_{s}\right)$ increases for the optimistic agent leading 
to a decrease of the market price of risk. There is then a shift towards lower (resp. higher) market prices of risk following good (bad) news. Similarly, there is a shift towards lower (resp. higher) risk free rates following bad (good) news. This means that in the general setting, we retrieve locally the same type of results as in the model with constant parameters, i.e. the fact that good news decrease the market price of risk and increase the risk free rate.

\section{Extension: a Model with Irrational as well as Rational Agents}

The aim of this section is to consider to what extent our results on irrational traders are robust to the presence of rational traders on the markets. For this purpose, we consider a model that is analogous to the main model except that there are now three agents : Agent 1, overestimating the instantaneous expected growth rate by $\sigma \delta$, Agent 2 , underestimating the instantaneous expected growth rate by $\sigma \delta$ and Agent 3, rationally expecting the instantaneous growth rate $\mu$. We suppose that the three agents have the same initial endowment $(1 / 3) e^{*}$. The individual consumption shares are given by $\tau_{i} \equiv \frac{c_{i}^{*}}{e^{*}}=\frac{M^{i}}{M^{1}+M^{2}+1}$, where $M^{3} \equiv 1$. The survival properties are different in this setting. Indeed, as shown in Yan (2008), it is easy to obtain in this setting that $\tau_{3}(t)$ converges to 1 almost surely, i.e. only the rational agent survives $^{14}$. This implies that instead of converging to an economy with two possible scenarios (one pessimistic scenario and one optimistic scenario), the economy converges to an economy with the rational scenario only. In particular, the instantaneous risk free rate converges to the rational risk free rate and the market price of risk converges to the rational market price of risk. The other results remain essentially true. The consumption shares $\tau_{1}(t)$ and $\tau_{2}(t)$ have the same distribution for all $t$, and none of the irrational agents eliminates the other one. The instantaneous prices are on average given by the rational prices, i.e. $E\left[M P R_{t}\right]=M P R(\operatorname{stdd})$ and $E\left[r_{t}^{f}\right]=r^{f}(\operatorname{stdd})$. We get that $\frac{\tau_{1}(t)}{\tau_{2}(t)}=\exp \left(2 \delta W_{t}\right)$, hence $\tau_{1}>\tau_{2}$ if and only if $\log e_{t}^{*}>E\left[\log e_{t}^{*}\right]$, i.e. in good states of the world.

\footnotetext{
${ }^{14}$ We have for $i=1,2, M_{t}^{i}=\exp \left[t\left(\delta_{i} \frac{W_{t}}{t}-\frac{\delta_{i}^{2}}{2}\right)\right]$ which converges to 0 when $t$ increases, since $\frac{W_{t}}{t} \rightarrow t \rightarrow \infty 0$
} 
This means that there is an optimistic bias (in terms of consumption shares) in the good states of the world and a pessimistic bias in the bad states of the world. We still get that the economy is dominated by the pessimistic agents in the very bad states of the world and dominated by the optimistic agents in the very good states of the world and that there is a shift in favor of the optimistic (resp. pessimistic) agents following a good (resp. a bad) shock. The market price of risk, which is given by $M P R_{t}=M P R(\operatorname{stdd})-\left(\tau_{1}(t)-\tau_{2}(t)\right) \delta$,

is countercyclical. The risk free rate, which is given by $r_{t}^{f}=r^{f}(\operatorname{stdd})+\sigma\left(\tau_{1}(t)-\tau_{2}(t)\right) \delta$ is procyclical. The yield curve still converges to the discount rate of the more pessimistic agent (even though the risk free rate converges to the rational rate). Finally, the state price density is still a mixture of lognormal distributions, which generates more variance.

\section{Conclusion}

In this paper we study the impact on the behavior of financial markets of irrational traders, when they are on average rational. We consider as our main model a model with logarithmic utility functions, two groups of agents, and constant biases but we show that the results pertain to more general power utility functions, to more than two groups of agents, to the presence of an additional group of rational investors and to more general models of disagreement.

To sum up, the model with unbiased disagreement is very different from the standard rational model, although they share common features. As in the standard setting, all agents survive. Moreover, at all date, the consumption shares have the same distribution. Finally, at all date, the prices remain on average the same as in the standard rational setting. These properties are consistent with the fact that disagreement is unbiased. However, the following features make the setting with unbiased disagreement very different from the rational setting and particularly interesting.

- There are waves of optimism and pessimism 
- The market price of risk is countercyclical (higher in recessions and lower in expansions)

- The risk free rate is procyclical .

- The market price of risk and the risk free rate are mean reverting.

- The yield curve is decreasing (at least in the long run).

- The long run risky asset's return is possibily higher, as is the long term risk premium.

- The state price density has greater variance.

- State price densities are mixtures of lognormal distributions.

- Volatility may be fluctuating.

We emphasize that the obtained properties only result from the (absolute) risk tolerance fluctuations and not on specific learning dynamics. Note also that we do not impose short sales constraints.

\section{References}

[1] Abel, A., 1989. Asset Prices under Heterogeneous Beliefs: Implications for the Equity Premium. Mimeo, University of Pennsylvania.

[2] Admati, A.R., 1985. A Noisy Rational Expectations Equilibrium for Multi-Asset Securities Markets. Econometrica, 53, 629-657.

[3] Aït-Sahalia, Y., and A.W. Lo, 1998. Nonparametric Estimation of State Price Densities Implicit in Financial Asset Prices. Journal of Finance, 53, 499-547.

[4] Basak, S., 2000. A Model of Dynamic Equilibrium Asset Pricing with Heterogeneous Beliefs and Extraneous Risk. Journal of Economic Dynamics and Control, 24, 63-95. 
[5] Berrada, T., 2006. Incomplete Information, Heterogeneity and Asset Pricing. Journal of Financial Econometrics, 4, 136-160.

[6] Biais, B., Bossaerts, P. and C. Spatt, 2009. Equilibrium Asset Pricing and Portfolio Choice under Asymmetric Information. To appear, Review of Financial Studies.

[7] Blanchard, O. and M. Watson, 1986. Are Business Cycles All Alike? In Robert Gordon, Ed. The American Business Cycle, 123-182. Chicago, University of Chicago Press.

[8] Blume, L., and D. Easley, 2006. If You're So Smart, Why Aren't You Rich ? Belief Selection in Complete and Incomplete Markets. Econometrica, 74, 929-966.

[9] Blume, L., and D. Easley, 2009. The Market Organism: Long Run Survival in Markets with Heterogeneous Traders. Journal of Economic Dynamics and Control, 33, 1023-1035.

[10] Cabrales, A., and T. Hoshi, 1996. Heterogeneous Beliefs, Wealth Accumulation, and Asset Price Dynamics. Journal of Economic Dynamics and Control, 20, 1073-1100.

[11] Calvet, L., Grandmont, J.-M., and I. Lemaire, 2002. Aggregation of Heterogeneous Beliefs and Asset Pricing in Complete Financial Markets. Working Paper.

[12] Campbell J.Y. and J.H. Cochrane, 1999. By Force of Habit: A Consumption-Based Explanation of Aggregate Stock Market Behavior. Journal of Political Economy, 107, 205-251.

[13] Chateauneuf, A., and M. Cohen, 1994. Risk Seeking with Diminishing Marginal Utility in a Nonexpected Utility Model. Journal of Risk and Insurance, 9, 77-91.

[14] Cochrane, J., 1991. Volatility Tests and Efficient Markets, a Review Essay. Journal of Monetary Economics, 27, 463-485.

[15] David, A., 2008. Heterogeneous Beliefs, Speculation, and the Equity Premium. Journal of Finance, 63, 41-83. 
[16] David, A., and P. Veronesi, 2002. Option Prices with Uncertain Fundamentals. CRSP Working Paper, University of Chicago.

[17] DeMarzo, P. and C. Skiadas, 1998. Aggregation, Determinacy, and Informational Efficiency for a Class of Economies with Asymmetric Information. Journal of Economic Theory, 80, 123-152.

[18] Detemple J., and S. Murthy, 1994. Intertemporal Asset Pricing with Heterogeneous Beliefs. Journal of Economic Theory, 62, 294-320.

[19] Diecidue, E., and P. Wakker, 2001. On the Intuition of Rank Dependent Utility. Journal of Risk and Insurance, 281-298.

[20] Duchin, R. and M. Levy, 2009. Disagreement, Portfolio Optimization and Excess Volatility. To appear, Journal of Financial and Quantitative Analysis.

[21] Dumas, B., Kurshev A., and R. Uppal, 2009. Equilibrium Portfolio Strategies in the Presence of Sentiment Risk and Excess Volatility. To appear, Journal of Finance.

[22] Fama, E. and K. French, 1988. Dividend Yields and Expected Stock Returns. Journal of Financial Economics, 22, 3-25.

[23] Fama, E. and K. French, 2008. Disagreement, Tastes and Asset Prices. CRSP Working Paper No. 552

[24] Friedman, B., 1986. Money, Credit and Interest Rates in the Business Cycle. In Robert Gordon, ed. The American Business Cycle, 123-182. Chicago, University of Chicago Press.

[25] Friedman, M., 1953. The case for flexible exchange rates. Essays in Positive Economics, University of Chicago Press, Chicago.

[26] Gollier, C., 2007. Whom Should We Believe ? Aggregation and Heterogeneous Beliefs. Journal of Risk and Uncertainty, 35, 107-127. 
[27] Hansen, L.P. and R. Jagannathan, 1991. Implications of Security Market Data for Models of Dynamic Economies. Journal of Political Economy, 99, 225-262.

[28] Hansen, L.P. and R. Jagannathan, 1997. Assessing Specification Errors in Stochastic Discount Factors Models. The Journal of Finance, 52, 557-590.

[29] Jackwerth, J.C., and M. Rubinstein, 1996. Recovering Probability Distributions from Asset Prices. Journal of Finance, 51, 1611-1631.

[30] Jouini, E. , and C. Napp, 2007. Consensus Consumer and Intertemporal Asset pricing with Heterogeneous Beliefs. Review of Economic Studies, 74, 1149-1174.

[31] Jouini, E. , Marin, J.-M. and C. Napp, 2008. Discounting and Divergence of Opinion. To appear, Journal of Economic Theory.

[32] Kogan, L., Ross, S., Wang, J., and M. Westerfield, 2006. The Price Impact and Survival of Irrational Traders. Journal of Finance, 61, 195-229.

[33] Kogan, L., Ross, S., Wang, J., and M. Westerfield, 2008. Market Selection. Working Paper.

[34] Levy, H., Levy, M. and G. Benita, 2006. Capital Asset Prices with Heterogeneous Beliefs. Journal of Business, 79, 1317-1354.

[35] Li, T., 2007. Heterogeneous Beliefs, Asset Prices, and Volatility in a Pure Exchange Economy. Journal of Economic Dynamics and Control, 31, 1697-1727.

[36] Mehra, R. and E. Prescott, 1985. The Equity Premium Puzzle. Journal of Monetary Economics, 15, 145-161.

[37] Merton, R., 1980. On Estimating the Expected Return on the Market: an Exploratory Investigation. Journal of Financial Economics, 8, 323-362. 
[38] Rubinstein, M., 1976. The Valuation of Uncertain Income Streams and the Pricing of Options. Bell Journal of Economics, 7, 407-425.

[39] Sandroni, A., 2000. Do Markets Favor Agents Able to Make Accurate Predictions ? Econometrica, 68, 1303-1334.

[40] Scheinkman, J., and W. Xiong, 2003. Overconfidence and Speculative Bubbles. Journal of Political Economy, 111, 1183-1219.

[41] Yaari, M., 1987. The Dual Theory of Choice under Risk. Econometrica, 55, 95-115.

[42] Yan, H., 2008a. Natural Selection in Financial Markets: Does It Work ? Management Science, 54, 1935-1950.

[43] Yan, H., 2008b. Is Noise Trading Cancelled out by Aggregation? Working Paper, Yale School of Management.

[44] Zapatero, F., 1998. Effects of Financial Innovations on Market Volatility when Beliefs are Heterogeneous. Journal of Economic Dynamics and Control, 22, 597-626. 


\section{APPENDIX I}

\section{Proof of Proposition 3.1}

1. According to Equation (3.1), we have $\tau_{1}=\frac{\left(M_{t}^{1} / M_{t}^{2}\right)}{1+\left(M_{t}^{1} / M_{t}^{2}\right)}$ hence $\tau_{1}(t)=\frac{\exp \left(2 \delta W_{t}\right)}{1+\exp \left(2 \delta W_{t}\right)}$ and $\tau_{2}(t)=\frac{\exp \left(-2 \delta W_{t}\right)}{1+\exp \left(-2 \delta W_{t}\right)}$ with $W_{t} \sim \mathcal{N}(0, t)$. The consumption shares have then the same distribution and it is symmetric with respect to $1 / 2$; in particular, we have $E\left[\tau_{i}(t)\right]=1 / 2$.

2. According to Equation (3.1), we have $\tau_{i}=\frac{1}{2} \frac{M^{i}}{M}$ with $M \equiv \frac{1}{2}\left(M^{1}+M^{2}\right)$. Agent $i$ has a larger share of aggregate consumption when $M^{i} \geq M$, i.e. in the states of the world that she overweights. Since $\tau_{1}(t)=\frac{\exp \left(2 \delta W_{t}\right)}{1+\exp \left(2 \delta W_{t}\right)}$, we have $\tau_{1}(t)=f\left(W_{t}\right)$ with $f(x)=\frac{\exp (2 \delta x)}{1+\exp (2 \delta x)}$. It is immediate that $f$ is increasing with $\lim _{-\infty} f=0$ and $\lim _{+\infty} f=1$.

3. We have $\tau_{1}(t)=f\left(W_{t}\right)$ with $f(x)=\frac{\exp (2 \delta x)}{1+\exp (2 \delta x)}$. Itô's Lemma and elementary algebra give the following Stochastic Differential Equations

$$
\begin{aligned}
d \tau_{1}(t) & =2 \delta^{2} \tau_{1}\left(1-\tau_{1}\right)\left(1-2 \tau_{1}\right) d t+2 \delta \tau_{1}\left(1-\tau_{1}\right) d W_{t} \\
& =2 \delta \tau_{1} \tau_{2}\left[\delta\left(\tau_{2}-\tau_{1}\right) d t+d W_{t}\right] \\
d \tau_{2}(t) & =-2 \delta \tau_{1} \tau_{2}\left[\delta\left(\tau_{2}-\tau_{1}\right) d t+d W_{t}\right] .
\end{aligned}
$$

It is immediate that the drift of $\tau_{1}$ is positive for $\tau_{1}<\frac{1}{2}$ and negative for $\tau_{1}>\frac{1}{2}$. The process $\tau_{1}$ exhibits then mean reversion around $\frac{1}{2}$.

\section{Proof of Proposition 3.2}

1. Since $q^{*}=(\exp (-\rho t))\left(\frac{1}{2} M^{1}+\frac{1}{2} M^{2}\right)\left(e^{*}\right)^{-1}, r_{f}=-\mu_{q^{*}}$ and $M P R=-\sigma_{q^{*}}$, we obtain through Itô's Lemma

$$
\begin{aligned}
r_{t}^{f} & =\rho+\mu-\sigma^{2}+\sigma\left(\tau_{1}(t) \delta-\tau_{2}(t) \delta\right), \\
M P R_{t} & =\sigma-\left(\tau_{1}(t)-\tau_{2}(t)\right) \delta .
\end{aligned}
$$

2. According to Equation (3.4), the market price of risk is lower in states of the world where $\tau_{1}(t)>\tau_{2}(t)$ and decreases with $\tau_{1}(t)$. Proposition 3.1 concludes. 
3. According to Equation (3.3), the risk free rate is higher in states of the world where $\tau_{1}(t)>\tau_{2}(t)$ and increases with $\tau_{1}(t)$. Proposition 3.1 concludes.

4. This is a direct consequence of Equation (3.4) and of Point 3 of Proposition 3.1.

\section{Proof of Proposition 3.3}

1. We can apply Jouini et al. (2008) to our specific setting by taking $\rho^{i}=\rho, \delta_{1}=\delta$ and $\delta_{2}=-\delta$. We rederive this result here for the sake of completeness. We have

$$
R_{t}=-\frac{1}{t} \log E\left[\left(\frac{1}{2} M_{t}^{1}+\frac{1}{2} M_{t}^{2}\right) \exp (-\rho t)\left(e_{t}^{*}\right)^{-1}\right]
$$

We then have $R_{t}=\rho+\mu-\sigma^{2}-\frac{1}{t} \log \left[\frac{1}{2} \exp (-\delta \sigma t)+\frac{1}{2} \exp (+\delta \sigma t)\right]$. The rest is immediate.

2. Let $\mu_{S}(s t d d) \equiv \rho+\mu-\sigma^{2}+\sigma \sigma_{S}$. We have $\mu_{S}(t)=\mu_{S}(s t d d)+\delta\left(\sigma-\sigma_{S}\right)\left(\tau_{1}-\tau_{2}\right)$ and

$$
\frac{1}{T} \log E\left[S_{T}\right]=\frac{1}{T} \log E\left[\exp \left\{\int_{0}^{T}\left(\mu_{S}(t)-\frac{\sigma_{S}^{2}}{2}\right) d t+\sigma_{S} W_{T}\right\}\right]
$$

Let us first prove that $\frac{1}{T} \log E\left[S_{T}\right] \leq \mu_{S}(s t d d)+\delta\left(\sigma-\sigma_{S}\right)$. Since by hypothesis $\delta\left(\sigma-\sigma_{S}\right) \geq$ 0 , we have $\mu_{S}(t) \leq \mu_{S}(s t d d)+\delta\left(\sigma-\sigma_{S}\right)$, hence

$$
\begin{aligned}
\frac{1}{T} \log E\left[S_{T}\right] & \leq \frac{1}{T} \log E\left[\exp \left\{\left(\int_{0}^{T} \mu_{S}(s t d d)+\delta\left(\sigma-\sigma_{S}\right)-\frac{\sigma_{S}^{2}}{2}\right) d t+\sigma_{S} W_{T}\right\}\right] \\
& \leq \mu_{S}(s t d d)+\delta\left(\sigma-\sigma_{S}\right) .
\end{aligned}
$$

We have by Equation (6.1)

$$
\begin{aligned}
& \frac{1}{T} \log E\left[S_{T}\right] \\
= & \mu_{S}(s t d d)+\frac{1}{T} \log E\left[\exp \left\{\int_{0}^{T}\left(\delta\left(\sigma-\sigma_{S}\right)\left(\tau_{1}-\tau_{2}\right)-\frac{\sigma_{S}^{2}}{2}\right) d t+\sigma_{S} W_{T}\right\}\right] \\
= & \mu_{S}(s t d d)+\frac{1}{T} \log E\left[\exp \left\{\int_{0}^{T}\left(\delta\left(\sigma-\sigma_{S}\right) \frac{\exp \left(2 \delta W_{t}\right)-1}{\exp \left(2 \delta W_{t}\right)+1}-\frac{\sigma_{S}^{2}}{2}\right) d t+\sigma_{S} W_{T}\right\}\right] .
\end{aligned}
$$


We introduce the probability measure $\bar{P}$ such that $\left.\frac{d \bar{P}}{d P}\right|_{F_{t}}=\exp \left(\sigma_{S} W_{t}-\frac{\sigma_{S}^{2}}{2} t\right)$. We have

$$
\frac{1}{T} \log E\left[S_{T}\right]=\mu_{S}(s t d d)+\frac{1}{T} \log E^{\bar{P}}\left[\exp \left\{\int_{0}^{T}\left(\delta\left(\sigma-\sigma_{S}\right) \frac{\exp \left(2 \delta W_{t}\right)-1}{\exp \left(2 \delta W_{t}\right)+1}\right) d t\right\}\right]
$$

Letting $\bar{W}_{t} \equiv W-\sigma_{S}$, we know that $\bar{W}$ is a Brownian motion under $\bar{P}$ and we obtain

$$
\begin{aligned}
& \frac{1}{T} \log E\left[S_{T}\right] \\
= & \mu_{S}(s t d d)+\frac{1}{T} \log E^{\bar{P}}\left[\exp \left\{\int_{0}^{T}\left(\delta\left(\sigma-\sigma_{S}\right) \frac{\exp \left(2 \delta \bar{W}_{t}+2 \delta \sigma_{S} t\right)-1}{\exp \left(2 \delta \bar{W}_{t}+2 \delta \sigma_{S} t\right)+1}\right) d t\right\}\right] \\
= & \mu_{S}(s t d d)+\frac{1}{T} \log E\left[\exp \left\{\int_{0}^{T}\left(\delta\left(\sigma-\sigma_{S}\right) \frac{\exp \left(2 \delta W_{t}+2 \delta \sigma_{S} t\right)-1}{\exp \left(2 \delta W_{t}+2 \delta \sigma_{S} t\right)+1}\right) d t\right\}\right] .
\end{aligned}
$$

We let $Y_{t} \equiv \frac{\exp \left(2 \delta W_{t}+2 \delta \sigma_{S} t\right)-1}{\exp \left(2 \delta W_{t}+2 \delta \sigma_{S} t\right)+1}$. We want to show that $\liminf \frac{1}{T} \log E\left[S_{T}\right] \geq \mu_{S}(s t d d)+$ $\delta\left(\sigma-\sigma_{S}\right)$ or equivalently that liminf $E\left[\exp \left\{\delta\left(\sigma-\sigma_{S}\right) N\left(\frac{1}{N} \int_{0}^{N} Y_{t} d t\right)\right\}\right]^{1 / N} \geq \exp \left(\delta\left(\sigma-\sigma_{S}\right)\right)$.

By Hölder's Inequality, we have $E\left[\exp \left\{\delta\left(\sigma-\sigma_{S}\right) N\left(\frac{1}{N} \int_{0}^{N} Y_{t} d t\right)\right\}\right]^{1 / N} \geq E\left[\exp \left\{\delta\left(\sigma-\sigma_{S}\right)\left(\frac{1}{N} \int_{0}^{N} Y_{t} d t\right.\right.\right.$ The quantity $Y_{t}=\frac{\exp t\left(2 \delta \frac{W_{t}}{t}+2 \delta \sigma_{S}\right)-1}{\exp t\left(2 \delta \frac{W_{t}}{t}+2 \delta \sigma_{S}\right)+1}$ converges almost surely towards 1 since $\frac{W_{t}}{t}$ converges towards 0 almost surely. Furthermore $Y_{t}$ is bounded. By Césaro's Lemma, we have that $\frac{1}{N} \int_{0}^{N} Y_{t} d t$ also converges almost surely towards 1 , hence $\exp \left\{\delta\left(\sigma-\sigma_{S}\right)\left(\frac{1}{N} \int_{0}^{N} Y_{t} d t\right)\right\}$ converges almost surely to $\exp \left(\delta\left(\sigma-\sigma_{S}\right)\right)$. Now, by Fatou's Lemma, we have

$\liminf E\left[\exp \left\{\delta\left(\sigma-\sigma_{S}\right)\left(\frac{1}{N} \int_{0}^{N} Y_{t} d t\right)\right\}\right] \geq E\left[\liminf \exp \left\{\delta\left(\sigma-\sigma_{S}\right)\left(\frac{1}{N} \int_{0}^{N} Y_{t} d t\right)\right\}\right]$ hence $\lim \inf E\left[\exp \left\{\delta\left(\sigma-\sigma_{S}\right)\left(\frac{1}{N} \int_{0}^{N} Y_{t} d t\right)\right\}\right] \geq \exp \left(\delta\left(\sigma-\sigma_{S}\right)\right)$ and $\liminf \frac{1}{T} \log E\left[S_{T}\right] \geq$ $\mu_{S}(s t d d)+\delta\left(\sigma-\sigma_{S}\right)$.

3. Immediate according to 1 . and 2 .

\section{APPENDIX II}

\section{Results for Power Utility Functions}

\section{A. Existence Result}


For general $\eta$, if $(1-\eta)(\mu-\sigma \delta)-\frac{1}{2} \frac{1}{\eta}(1-\eta) \sigma^{2}>0$, then there exists an equilibrium $\left(q^{*},\left(c_{i}^{*}\right)_{i=1,2}\right)$ given by

$$
c_{i}^{*}=\left(\frac{\gamma_{i}\left(M^{i}\right)^{\eta}}{\gamma_{1}\left(M^{1}\right)^{\eta}+\gamma_{2}\left(M^{2}\right)^{\eta}}\right) e^{*}, \quad q^{*}=\left(\gamma_{1}\left(M^{1}\right)^{\eta}+\gamma_{2}\left(M^{2}\right)^{\eta}\right)^{1 / \eta}\left(e^{*}\right)^{-1 / \eta}
$$

for some positive constants $\left(\gamma_{i}\right)$ with $\gamma_{1}+\gamma_{2}=1$. For $\eta=1 / 2$, we have $\frac{\gamma_{1}}{\gamma_{2}}=\sqrt{\frac{\mu-\sigma^{2}+\sigma \delta}{\mu-\sigma^{2}-\sigma \delta}}$. More generally, for $\eta<1$, there is a small bias towards the optimistic investor $\left(\gamma_{1}>1 / 2\right)$, and for $\eta>1$, there is a small bias towards the pessimistic investor $\left(\gamma_{2}>1 / 2\right)$.

\section{B. Distribution of Consumption Shares}

1. The random variables $\tau_{i}(t)$ have the following density functions on $[0,1]$

$$
f_{\tau_{i}(t)}(x)=\frac{1}{2 \sqrt{2 \pi t} \eta \delta} \frac{1}{x(1-x)} \exp -\frac{1}{2} \frac{\left[\log \left(\frac{x}{1-x}\right)-\log \left(\frac{\gamma_{i}}{\gamma_{j}}\right)\right]^{2}}{4 \eta^{2} \delta^{2} t} \mathbf{1}_{[0,1]}(x)
$$

2. For all $t$ and for $i=1,2$, the median of the distribution of the consumption share of each agent is given by her initial "biased wealth level" $\gamma_{i}$, i.e., we have $P\left(\tau_{i}(t)>\gamma_{i}\right)=$ $P\left(\tau_{i}(t)<\gamma_{i}\right)=1 / 2$.

\section{Consumption Shares Stochastic Processes}

1. The optimal consumption shares are given by $\tau_{i}=\frac{\gamma_{i}\left(M^{i}\right)^{\eta}}{\gamma_{1}\left(M^{1}\right)^{\eta}+\gamma_{2}\left(M^{2}\right)^{\eta}}$. Each agent has a larger share of aggregate endowment in the states that she thinks more probable. The share $\tau_{1}$ is an increasing function of $W$.

2. The stochastic processes $\tau_{i}(t)$ satisfy the following SDE

$$
d \tau_{i}(t)=2 \eta \delta \tau_{i} \tau_{j}\left[\eta \delta\left(\tau_{j}-\tau_{i}\right) d t+d W_{t}\right]
$$

\section{Asset Pricing}


1. The instantaneous risk free rate and the market price of risk are given by

$$
\begin{aligned}
r_{t}^{f} & =r_{t}^{f}(\text { stdd })+\frac{\sigma}{\eta}\left(\tau_{1}(t)-\tau_{2}(t)\right) \delta-2(\eta-1) \delta^{2} \tau_{1}(t) \tau_{2}(t), \\
M P R_{t} & =M P R_{t}(\text { stdd })-\left(\tau_{1}(t)-\tau_{2}(t)\right) \delta
\end{aligned}
$$

where $r_{t}^{f}($ stdd $)=\frac{\mu}{\eta}-\frac{\sigma^{2}}{2}\left(1+\frac{1}{\eta}\right)$ and $M P R($ stdd $)=\frac{\sigma}{\eta}$. The quantity $\tau_{1}(t) \tau_{2}(t)$ converges to 0 in Probability.

2. The market price of risk is lower in states of the world where $\tau_{1}(t)>\tau_{2}(t)$, i.e. in "good" states of the world and higher in states of the world where $\tau_{2}(t)>\tau_{1}(t)$, i.e. in "bad" states of the world. The risk free rate is higher in "very good" states of the world and lower in "very bad" states of the world.

3. The market price of risk exhibits mean reversion with

$$
M P R_{t}=\frac{\sigma}{\eta}-\delta\left(\tau_{1}(t)-\tau_{2}(t)\right)=\frac{\sigma}{\eta}-\delta\left(2 \tau_{1}(t)-1\right)
$$

4. The discount rate is always converging to the pessimistic rate in the long run. For $\eta=1 / 2$, we have for all $t$,

$$
R_{t}=2 \mu-3 \sigma^{2}-\frac{1}{t} \log \left[\gamma_{1}^{2} \exp -(2 \delta \sigma t)+\gamma_{2}^{2} \exp (2 \delta \sigma t)+2 \gamma_{1} \gamma_{2} \exp -\frac{1}{2} \delta^{2} t\right]
$$

and $R_{\infty}=2 \mu-3 \sigma^{2}-2 \sigma \delta$.

\section{E. Volatility}

We consider a stock $\mathrm{S}$, whose dividends are the aggregate endowment process, $d S_{t}+e_{t}^{*} d t=$ $S_{t}\left[\mu_{s} d t+\sigma_{s} d W_{t}\right]$

We consider the case $\eta=1 / 2$. We suppose that $\rho=0$ and $\mu-\sigma \delta-\sigma^{2}>0$. We have in the standard rational setting $S_{t}=\frac{1}{\mu-\sigma^{2}} e_{t}^{*}$ and $\sigma_{S}=\sigma$. We denote by $S^{1}$ (resp. $S^{2}$ ) the prices that would prevail if the economy was made of the optimistic (resp. pessimistic) agents only. 
In the setting with disagreement, we get

$$
\begin{aligned}
S_{t} & =e_{t}^{*}\left[a \tau_{1}^{2}+b \tau_{2}^{2}+2 c \tau_{1} \tau_{2}\right] \\
\sigma_{S} & =\sigma+2 \delta \tau_{2}\left(1-\frac{b \tau_{2}+c \tau_{1}}{a \tau_{1}^{2}+b \tau_{2}^{2}+2 c \tau_{1} \tau_{2}}\right)
\end{aligned}
$$

with $a=\frac{1}{\mu+\delta \sigma-\sigma^{2}}, b=\frac{1}{\mu-\delta \sigma-\sigma^{2}}, c=\frac{1}{\mu+\frac{1}{2} \delta^{2}-\sigma^{2}}$. In particular, in the long run, $\frac{S_{t}}{e_{t}^{*}}$ either converges to $\frac{S_{t}^{1}}{e_{t}^{*}}$ or to $\frac{S_{t}^{2}}{e_{t}^{*}}$ with a probability 1 , and $\sigma_{S}$ converges to $\sigma$.

\section{Proof of E.}

We have

$$
\begin{aligned}
& S_{t} \\
= & \frac{\left(e_{t}^{*}\right)^{2}}{M_{t}} E_{t}\left[\int_{t}^{+\infty} M_{s}\left(e_{s}^{*}\right)^{-1} d s\right]=\frac{e_{t}^{*}}{M_{t}} E_{t}\left[\int_{t}^{+\infty}\left(\gamma_{1}^{2} M_{s}^{1}+\gamma_{2}^{2} M_{s}^{2}+2 \gamma_{1} \gamma_{2} \sqrt{M_{s}^{1} M_{s}^{2}}\right)\left(\frac{e_{s}^{*}}{e_{t}^{*}}\right)^{-1} d s\right] \\
= & e_{t}^{*}\left(\gamma_{1}^{2} \frac{M_{t}^{1}}{M_{t}}\right) E_{t}\left[\int_{t}^{+\infty}\left(\frac{M_{s}^{1}}{M_{t}^{1}}\right)\left(\frac{e_{s}^{*}}{e_{t}^{*}}\right)^{-1} d s\right] \\
& +e_{t}^{*}\left(\gamma_{2}^{2} \frac{M_{t}^{2}}{M_{t}}\right) E_{t}\left[\int_{t}^{+\infty}\left(\frac{M_{s}^{2}}{M_{t}^{2}}\right)\left(\frac{e_{s}^{*}}{e_{t}^{*}}\right)^{-1} d s\right] \\
& +e_{t}^{*}\left(2 \gamma_{1} \gamma_{2} \sqrt{\frac{M_{t}^{1}}{M_{t}}} \sqrt{\frac{M_{t}^{2}}{M_{t}}}\right) E_{t}\left[\int_{t}^{+\infty}\left(\sqrt{\frac{M_{s}^{1} M_{s}^{2}}{M_{t}^{1} M_{t}^{2}}}\right)\left(\frac{e_{s}^{*}}{e_{t}^{*}}\right)^{-1} d s\right] \\
= & e_{t}^{*}\left[\tau_{1}^{2}(t) a_{t}+\tau_{2}^{2}(t) b_{t}+2 \tau_{1}(t) \tau_{2}(t) c_{t}\right]
\end{aligned}
$$

with $a_{t}=E_{t}\left[\int_{t}^{+\infty}\left(\frac{M_{s}^{1}}{M_{t}^{1}}\right)\left(\frac{e_{s}^{*}}{e_{t}^{*}}\right)^{-1} d s\right], b_{t}=E_{t}\left[\int_{t}^{+\infty}\left(\frac{M_{s}^{2}}{M_{t}^{2}}\right)\left(\frac{e_{s}^{*}}{e_{t}^{*}}\right)^{-1} d s\right]$ and $c_{t}=E_{t}\left[\int_{t}^{+\infty}\left(\sqrt{\frac{M_{s}^{1} M_{s}^{2}}{M_{t}^{1} M_{t}^{2}}}\right)\left(\frac{e_{s}^{*}}{e_{t}^{*}}\right)^{-1}\right.$ Elementary algebra yields $a_{t}=\frac{1}{\mu+\delta \sigma-\sigma^{2}}, b_{t}=\frac{1}{\mu-\delta \sigma-\sigma^{2}}, c_{t}=\frac{1}{\mu+\frac{1}{2} \delta^{2}-\sigma^{2}}$.

\section{Results for more general models of disagreement}

We have $\tau_{1}(t)=f\left(X_{t}\right)$ with $f(x)=\frac{\exp x}{1+\exp x}$ and $X_{t}=\int_{0}^{t} 2 \delta(s, \omega) d W_{s}$. By Itô's Lemma 
and elementary algebra, we get the following Stochastic Differential Equations

$$
\begin{aligned}
d \tau_{1}(t) & =2 \delta^{2} \tau_{1}\left(1-\tau_{1}\right)\left(1-2 \tau_{1}\right) d t+2 \delta \tau_{1}\left(1-\tau_{1}\right) d W_{t} \\
& =2 \delta \tau_{1} \tau_{2}\left[\delta\left(\tau_{2}-\tau_{1}\right) d t+d W_{t}\right] \\
d \tau_{2}(t) & =-2 \delta \tau_{1} \tau_{2}\left[\delta\left(\tau_{2}-\tau_{1}\right) d t+d W_{t}\right]
\end{aligned}
$$

